\title{
A Distributed Channel Access Scheduling Scheme with Clean-Air Spatial Reuse for Wireless Mesh Networks*
}

\author{
Yuan-Chieh Lin ${ }^{1}$, Shun-Wen Hsiao ${ }^{1}$, Li-Ping Tung ${ }^{2}$, Yeali S. Sun ${ }^{1}$, \\ and Meng Chang Chen ${ }^{2}$ \\ ${ }^{1}$ Dept. of Information Management, National Taiwan University, Taipei, Taiwan \\ \{r94001, r93011, sunny\}@im.ntu.edu.tw \\ ${ }^{2}$ Institute of Information Science, Academia Sinica, Taipei, Taiwan \\ \{lptung, mcc\}@iis.sinica.edu.tw
}

\begin{abstract}
There are two effective approaches to maximize network capacity throughput: increasing concurrent non-interfering transmissions by exploiting spatial reuse and increasing transmission rates of active senders. These two ways are, however, a tradeoff due to the signal interference. In this paper, we propose a distributed channel access scheduling scheme under a Clean-Air Spatial Reuse architecture which spans both the MAC layer and the network planning plane to scale a wireless mesh network to high network capacity throughput and large coverage. Simulations results of the network capacity throughput performance under different levels of Clean-Air Spatial Reuse policies are presented. The results show that having more number of concurrent transmission pairs scheduled in each time slot usually can compensate the negative effect of using lower transmission rates of transmission links and result in better throughput performance.
\end{abstract}

\section{Introduction}

The wireless mesh network is expected to provide large transmission capacity and serve as a promising complementary solution to existing broadband access infrastructure. An important performance metric to evaluate the effectiveness of such a network is the network capacity throughput defined as the aggregate number of bits that can be successfully received by designated receivers within the network for a certain period of time.

The main factor that limits the network capacity throughput of a wireless mesh network is the interference between neighboring nodes when using a shared medium [1]. The network capacity throughput of a wireless mesh network is determined by the number of non-interfering transmissions that can be achieved at the same time (i.e., transmission concurrency) and their transmission rates. According to the Shannon's Channel Capacity Theorem [2] and the SINR definition, having a successful transmission with higher transmission rate at the sender requires higher SINR at the

This research is partly supported by National Science Council under grants NSC 95-2221-E-001-018-MY3 and NSC 95-2221-E-002-192. 
receiver which means it would be better to keep the interference low at the receiver by controlling the number of concurrent transmissions in the neighborhood. Conversely, a successful transmission can also be achieved by lowering a sender's transmission rate in a lower SINR environment which allows more number of concurrent non-interfering transmissions in the neighborhood. In other words, transmission concurrency and senders' transmission rates are indeed a tradeoff. Fig. 1 shows the network capacity throughputs under two different transmission rate and scheduling schemes. Note that the amount of increases between transmission rate and SINR has the logarithm relationship. Hence, to accomplish higher network capacity throughput, it would be more effective to do linear increase of the number of concurrent transmissions and $\log$-scale decrease of the transmission rates of individual links.

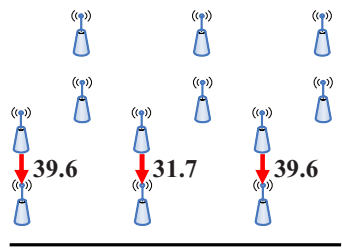

(a) $\quad 110.9$ Mbps

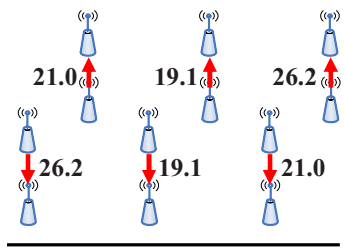

(b) $132.6 \mathrm{Mbps}$

Fig. 1. Network capacity throughput comparison under two different transmission rates and scheduling schemes (link bandwidth $\mathrm{W}=10 \mathrm{Mhz}$, path loss exponent $=4.0$ )

There are a number of works in the past studying the problem of how concurrent non-interfering transmissions in a TDMA-based mesh network may affect the arrangement of individual link transmission rate and the resulting network capacity throughput. Most of the work use some techniques such as compatibility matrix [3] to first find non-interfering transmission pairs and then use centralized algorithms for time slot assignment[4][5][6][7]. Various linear programming and heuristic methods are proposed for time slot assignment [8][1]. These works require prior knowledge of the traffic requirements and the interference relationship between wireless nodes, and assume they do not change frequently. The other drawbacks for completely centralized access scheduling in mesh networks include optimal and near-optimum scheduling are highly complex and time consuming; and it needs global information of the traffic demands in the network. This approach may not be computationally efficient for real-time multi-access network scheduling.

Due to inherent complexities of the interference in a mesh network and without global knowledge of the traffic demand, realizing a practical distributed data transmission schedule raises great challenges in coordinating active transmission pairs to avoid collision. One solution is the election-based three-way handshake scheme in IEEE 802.16 networks [9]. This scheme incurs long connection setup delay even for small size mesh networks. In [10], they show that more than 50 control message exchanges are necessary to complete a single three-way handshake procedure in a 30-node network. In [11], a distributed algorithm is used to dynamically tune each wireless station's power and transmission rate to achieve optimal spatial reuse and network capacity throughput in a CSMA-based network. In [12], they investigate 
receiver's sensitivity to different transmission rate, transmission range, node topology, and SINR in determining the optimal carrier sense range for a CSMA-based network. These results can not be directly applied to TDMA-based mesh networks.

In this work, our design goals are two folds. First, we wish to retain the flexibility of distributed channel access scheduling but reduce the complexity of distributed multi-access contention resolution. Second, we want to increase transmission concurrency as possible in the control/data slot access to raise network capacity throughput by exploiting the spatial reuse property and with the support of variable transmission rate capability at wireless stations. Here, we propose a combined approach which spans both the MAC layer and the network planning plane to scale a wireless mesh network to high network capacity throughput and large coverage. We use a regular hexagonal wireless mesh network (as shown in Fig. 2) as an illustrative example throughout the paper. However, we emphasize that the approach is general and applicable to wireless mesh networks with any arbitrary topology.

The rest of the paper is organized as follows. In Section 2, we present the distributed three-way handshake protocol for dynamic data transfer schedule setup by active transmission pairs in the mesh network. Section 3 presents the proposed planning model. Section 4 describes the distributed dynamic data transfer scheduling scheme combined with the planning model. Section 5 presents the simulations results of the network capacity throughput performance. Section 6 gives the conclusion.

\section{The Three-Way Handshake Protocol for Distributed Data Packet Scheduling}

Assume the wireless network access time is synchronized and slotted. The time slots are organized into a sequence of frames. Each frame consists of a control subframe and a data subframe. The control subframe is comprised of a number of control opportunity round used by active sender-receiver pairs to establish data transfer connections. A round consists of three control slots for the three-way handshake. The data subframe consists of a number of fixed-size data slots. To reserve data slots, an active sender must first obtain a handshake opportunity to initiate the connection setup with the intended receiver and successfully agree upon each other with a feasible data slot transmission schedule (or reservation). An important function of this protocol is to let the sender and receiver's "neighbors" to lean their data slot reservation. When the neighbors engage their own data slot scheduling, they will exclude these reserved data slots in the negotiation to avoid collision.

Now, the design issue becomes how far the transmission range of the control messages should go or the scope of "neighbors". To have less number of concurrent transmissions at a data slot, the negotiation information needs to reach more number of nodes as possible, and vice versa.

\section{The Planning Model}

In this paper, a planning model is developed to $a$ ) reduce the complexity and overhead in control channel access scheduling; $b$ ) raise network capacity throughput by 
exploiting transmission concurrency for both the control and data channel accesses. There are four processes: the specification of the Level-N Clean-Air Spatial Reuse policy, the formation of the concurrent data access groups, the formation of the concurrent control access groups, and the determination of the transmission rate of the stations in each access group.

\subsection{The Level-N Clean-Air Spatial Reuse Policy}

There are $\mathrm{V}$ stations, denoted as $\mathrm{SS}_{1}, \mathrm{SS}_{2}, \ldots, \mathrm{SS}_{\mathrm{V}}$, in a TDMA-based mesh network. Each $\mathrm{SS}_{\mathrm{i}}$ has at most $\mathrm{k} \mathrm{SS}_{\mathrm{j}}$ 's within the distance $\mathrm{d}$ which are referred to as the level-1 neighbors. Note the distance $\mathrm{d}$ could be arbitrary and is restrained by the maximum transmission range. The level-1 neighbors of the station's level-1 neighbors are referred to as the level-2 neighbors, and so on (as shown in Fig. 2). The idea of defining Level-N Clean-Air Spatial Reuse policy is to specify the minimum scope (level) between any two concurrent transmissions in the network, hence the name clean air. The policy will be used to partition all possible sender-receiver pairs in the network into distinct logical concurrent access groups. Pairs within the same group are allowed to transmit at the same time slot. Hence, given the Level-N Clean-Air Spatial Reuse policy, the assignment of active sender-receiver pairs to an access group must satisfy the following two conditions:

- for every SS assigned to transmit in the slot, all its level-1, level-2, .., up to level-N neighbors, except for the intended receiver must not be scheduled for transmitting and receiving;

- for every SS scheduled to receive, all its level-1, level-2, .., up to level-N neighbors, except for the intended transmitter must not be scheduled for transmitting and receiving.

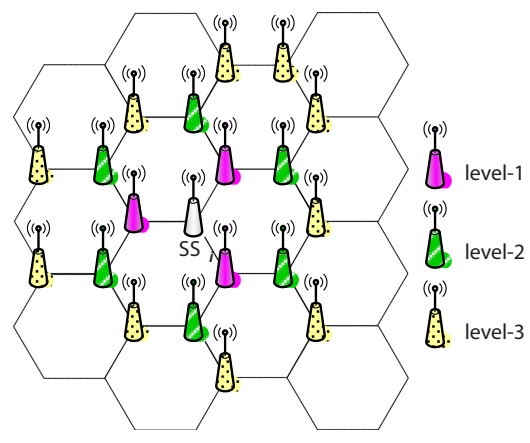

Fig. 2. The wireless mesh network and the Level-N relationship between $\mathrm{SS}_{\mathrm{i}}$ and its neighbors

To further increase the number of concurrent transmissions in a group, we also define the Level-0 Clean-Air Spatial Reuse policy with the following two conditions:

- for every SS scheduled to transmit, all its level-1 neighbors, except for the intended receiver must not be scheduled for receiving; 
- for every SS scheduled to receive, all its level-1 neighbors, except for the intended transmitter must not be scheduled for transmitting.

The formations of the concurrent data and control access groups will take the same Level-N policy to assure data schedule consistency of the stations in the network.

\subsection{The Concurrent Data Access Groups}

In this process, we partition all possible sender-receiver pairs in the network into a number of concurrent data access groups according to the specified Level-N Clean-Air Spatial Reuse policy. Pairs within the same group can be scheduled to transmit at the same data slot (Note that depending on the traffic demands, the proposed distributed data scheduling scheme in Section 4 allows pairs belonging to different groups to transmit at the same data slot). Assume all data transmissions occur between a station and one of its level-1 neighbors. We first divide all possible transmission pairs into a number of clusters. Then, the partitioning algorithm is applied to each cluster. It is a greedy algorithm that assigns as many sender-receiver pairs to the same access group as possible according to the Level-N Clean-Air Spatial Reuse policy constraint. Fig. 3 shows the initial six clusters which result in twelve concurrent data access groups for the Level-1 Clean-Air Spatial Reuse policy.

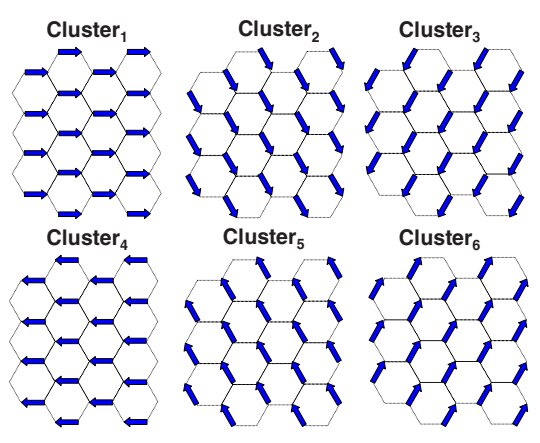

(a) initial 6 clusters

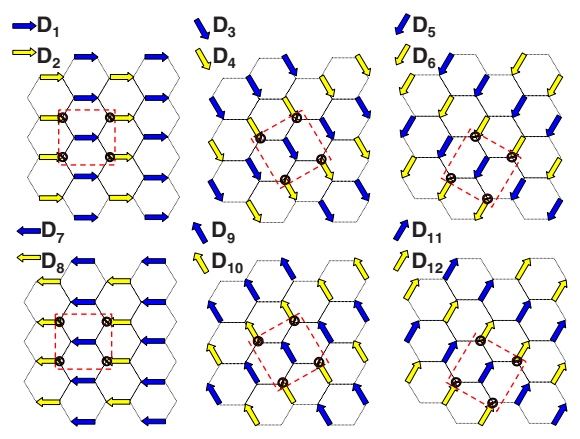

(b) 12 concurrent data access groups

Fig. 3. The greedy initial clustering and concurrent data access group assignment for the Level-1 Clean-Air Spatial Reuse policy

\subsection{The Concurrent Control Access Group}

To regulate the control subframe access, we partition all possible transmission pairs into a number of concurrent control access groups. For the sender-receiver pairs in the same group, they can do control message exchange at the control opportunity round designated to the group. Since within a control access group we must assure all sender-receiver pairs can successfully perform the three-way handshake protocol, the "clean-air" constraint must assure all their level-1, $\cdots$, up to level-N neighbors are 
non-busy. It has more stringent requirement of transmission concurrency than in the concurrent data access grouping.

\subsection{The Transmission Rate Assignment}

In this process, each sender of an access group is assigned a static and deterministic transmission rate so that all sender-receiver pairs in the same group can all transmit at the same time slot successfully. Here, we assume all sender-receiver pairs within the same group are active and stations transmit at the sufficiently large power level that satisfies receivers' capture threshold requirements at any achievable transmission rate. According to the adopted Clean-Air Spatial Reuse policy, we calculate individual receiver's SINR value and find the maximum achievable transmission rate for the sender.

\section{The Distributed Data Scheduling and Reservation Schemes}

Under the planning model, each station has the following knowledge: $a$ ) the Clean-Air Spatial Reuse policy employed; $b$ ) the concurrent control access group it belongs to; $c$ ) the concurrent data access group it belongs to. Meantime, it maintains a state vector recording the reservation status of the data subframe. Each element in the state vector has the value of "Free" (no one reserved or reserved by pairs of my group), "Busy" (already reserved by the other groups), "Send" (for my sending) or "Receive" (for my receiving). The number of slots of the control subframe is assumed to be equal to the number of the concurrent control access groups so that every station has a control message access opportunity on a periodic basis.

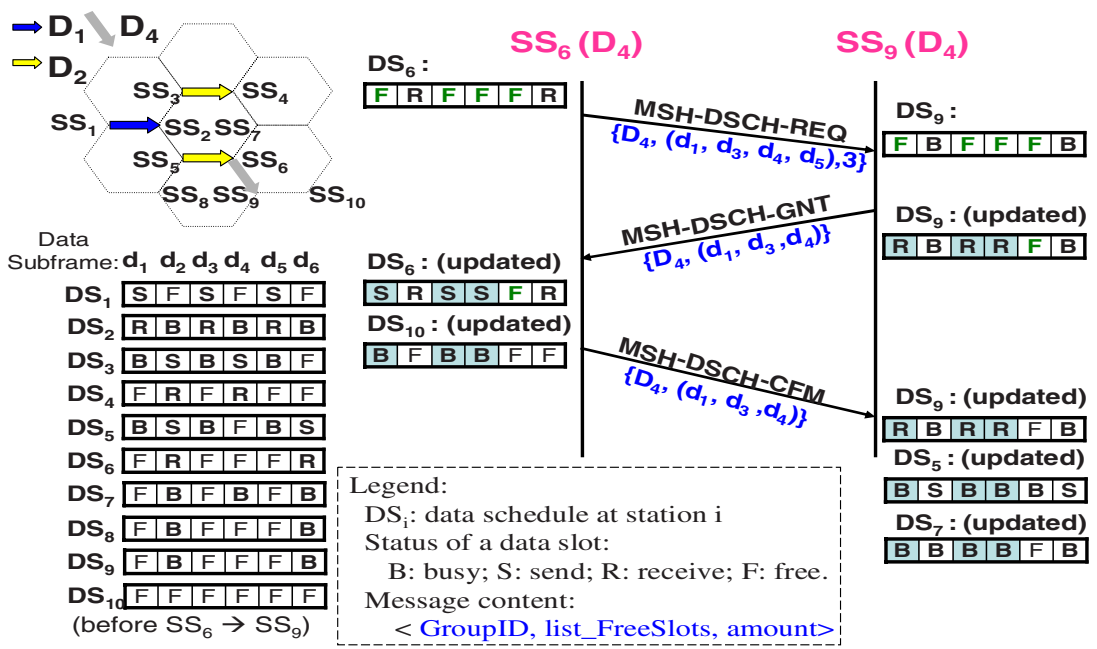

Fig. 4. The distributed data scheduling and reservation 
An example of distributed data scheduling and reservation under the Level-1 policy is depicted in Fig. 4. Suppose $S S_{6}$ wants to send three data packets to its Level-1 neighbor $S S_{9}$; they belong to the data access group $\mathrm{D}_{4}$ and control access group $\mathrm{C}_{4} . S S_{6}$ uses the opportunity round designated to $\mathrm{C}_{4}$ to negotiate a feasible data reservation schedule with $S S_{9}$. The figure shows the schedule exchange and negotiation between them. Meantime, their neighbors are required to update their state vectors accordingly. After establishing the data transfer schedule, $S S_{6}$ will send data packets to $S S_{9}$ on the reserved data slots. Here, $S S_{1}$ and $S S_{6}$ can transmit at the same time slots (i.e., $\mathrm{d}_{1}$ and $\mathrm{d}_{3}$ ) even though they belong to different data access group.

\section{Performance Evaluation}

In this section, we present the network capacity throughput performances under different Clean-Air Spatial Reuse policies in balancing between the number of concurrent transmissions and individual sender's transmission rate via simulation. Consider a mesh network with 24 nodes which are always backlogged. The frame duration is $10 \mathrm{~ms}$; there are 64 data slots in the data subframe; the bandwidth is $10 \mathrm{MHz}$. All simulations are implemented in $\mathrm{C}$. The transmission rate requirements are based on the data in [13]. For example, the minimum capture threshold is $1.9484(2.9 \mathrm{~dB})$ and the corresponding transmission rate is $10 \mathrm{Mbps}$.

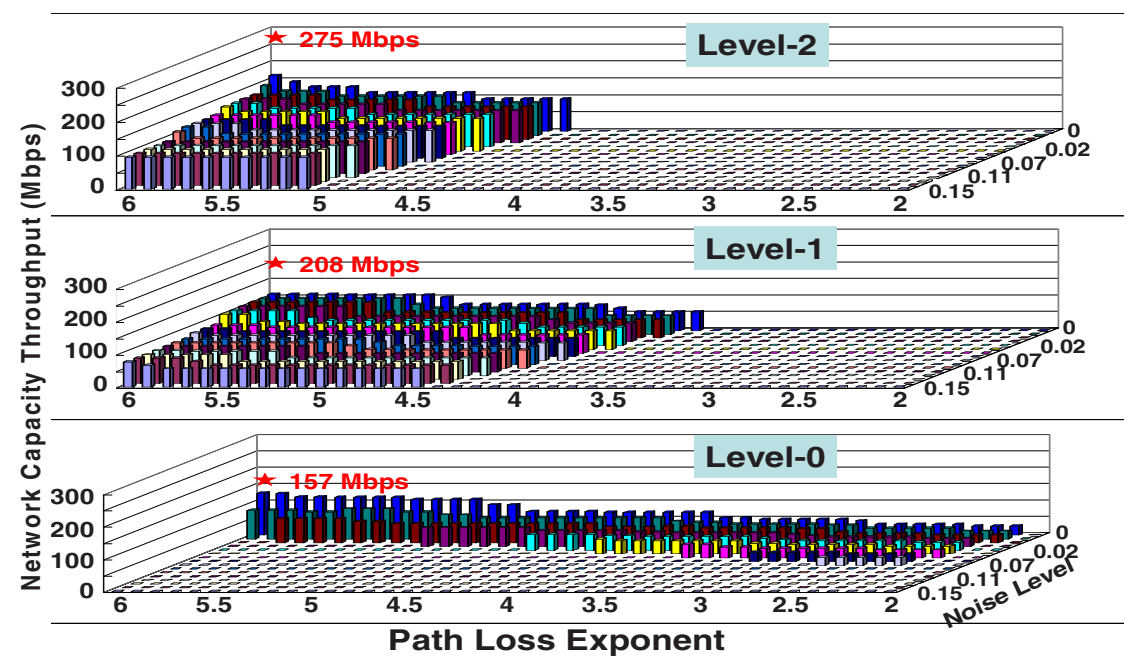

Fig. 5. The network capacity throughputs for Level-0, -1 and -2 Clean-Air Spatial Reuse policies under different environmental variables

Fig. 5 presents the network capacity throughputs for Level-0, -1 and -2 Clean-Air Spatial Reuse policies under different environmental conditions. The Level-0 policy achieves the highest network capacity throughput due to more number of concurrent 
transmission pairs are scheduled in each time slot which compensates the negative effect of using lower transmission rates. However, Level-0 policy may not have feasible scheduling solutions for environments with larger path loss exponent such as in metropolitan areas. The values with the start sign are the theoretical upper bounds of the throughput calculated according to the Shannon's Channel Capacity Theorem.

\section{Conclusions}

In this paper, we propose an approach to address the problem of channel access scheduling in wireless mesh networks to raise network capacity throughput. First, we propose a planning model with three key elements. The Level-N Clean-Air Spatial Reuse policy sets the minimum scope (level) of scheduling concurrent non-interfering transmission pairs in the wireless mesh network. Two algorithms are proposed to divide the network into distinct logical concurrent control/data access groups to raise network capacity throughput. Then each sender is assigned with a static transmission rate so that all sender-receiver pairs can have successfully transmissions. Based on the planning model, a distributed three-way handshake protocol is used for dynamic data scheduling. The combined scheme achieves the flexibility of dynamic and distributed channel access scheduling with reduced complexity (compared to pure distributed approach), and high network capacity throughput. The simulation results show that having more number of concurrent transmission pairs scheduled in each time slot usually can compensate the negative effect of using lower transmission rates and achieve better throughput performance.

\section{Reference}

1. Brar, G., Blough, D.M., Santi, P.: Computationally Efficient Scheduling with the Physical Interference Model for Throughput Improvement in Wireless Mesh Networks. In: ACM MobiCom, pp. 2-13 (2006)

2. Goldsmith, A.: Wireless Communications. Cambridge University Press (2005)

3. Nelson, R., Kleinrock, L.: Spatial TDMA: A Collision Free Multihop Channel Access Protocol. IEEE Transactions on Communications 33(9), 934-944 (1985)

4. Cheng, S.-M., Lin, P., Huang, D.-W., Yang, S.-R.: A Study on Distributed/Centralized Scheduling for Wireless Mesh Network. In: IWCMC, pp. 599-604 (2006)

5. Wei, H.-Y., Ganguly, S., Izmailov, R., Hass, Z.J.: Interference-Aware IEEE 802.16 WiMax Mesh Networks. In: IEEE VTC 2005-Spring, pp. 3102-3106 (2005)

6. Kabbani, A., Salonidis, T., Knightly, E.W.: Distributed Low-Complexity Maximum-Throughput Scheduler for Wireless Backhaul Networks. In: IEEE INFOCOM, pp. 2063-2071 (2007)

7. Chen, J., Chi, C., Guo, Q.: An Odd-Even Alternation Mechanism for Centralized Scheduling in WiMAX Mesh Network. In: GLOBECOM, pp. 1-6 (2006)

8. Viswanathan, H., Mukherjee, S.: Throughput-Range Tradeoff of Wireless Mesh Backhaul Networks. IEEE Journal on Selected Areas in Communications 24(3), 593-602 (2006)

9. IEEE Standard for Local and Metropolitan Area Networks Part 16: Air Interface for Fixed Broadband Wireless Access Systems. IEEE Std 802.16-2004 (2004) 
10. Cao, M., Ma, W., Zhang, Q., Wang, X., Zhu, W.: Modeling and Performance Analysis of the Distributed Scheduler in IEEE 802.16 Mesh Mode. In: ACM MobiHoc, pp. 78-89 (2005)

11. Kim, T.-S., Lim, H., Hou, J.C.: Improving Spatial Reuse through Tuning Transmit Power, Carrier Sense Threshold, and Data Rate in Multihop Wireless Network. In: ACM MobiCom, pp. 366-377 (2006)

12. Zhai, H., Fang, Y.: Physical Carrier Sensing and Spatial Reuse in Multirate and Multihop Wireless Ad Hoc Networks. In: IEEE INFOCOM, pp. 1-12 (2006)

13. WiMAX Forum, http://www.wimaxforum.org 